\title{
Comportamiento epidemiológico de la desnutrición en menores de 5 años, Colombia 2016-2019
}

\section{Epidemiological behavior of malnutrition in children under 5 years of age, Colombia 2016-2019 \\ Comportamento epidemiológico da desnutrição em crianças menores de 5 anos, Colômbia 2016-2019}

\author{
Mónica Paola Quemba-Mesa ${ }^{1}$ \\ Jhully Constanza Herrera-Tarapues ${ }^{2}$ \\ Alejandra Mendoza-Ortiz ${ }^{3}$ \\ Benigno Mendoza-Ortiz ${ }^{4}$
}

\begin{abstract}
Resumen
Objetivo: Analizar el comportamiento epidemiológico y la prevalencia de la desnutrición aguda en niños menores de 5 años en Colombia durante los años 2016 al 2019. Materiales $y$ métodos: Estudio cuantitativo - descriptivo de tipo retrospectivo. La población y muestra de análisis del presente estudio está conformada por 53.434 reportes consolidados en el Sistema integrado de información de la protección social SISPRO de niños y niñas menores de 5 años con desnutrición aguda en Colombia, disponibles entre los años 2016 a 2019. Resultados: En 2016 se presenta una tasa de mortalidad por desnutrición de 0.08 casos por cada 10.000 habitantes en contraste con el 2019 donde esta tasa aumento a 0,42; en el 2016 se presenta una tasa de morbilidad por desnutrición aguda de 22,4 casos por cada 10.000 habitantes en contraste con el 2019 donde esta tasa aumentó a 40,5. Los años de vida perdidos por mortalidad prematura oscilaron entre 1.162 en el 2016 a 6.411 en el 2019 y los años vividos con discapacidad oscilaron entre 588 en 2016 a 2.257 en 2019; los años de vida ajustados por discapacidad oscilaron entre 2.402 en 2016 a 8.668 en el 2019. Conclusiones: Es necesario el fortalecimiento de las intervenciones desde las políticas sociales enfocadas en los determinantes sociales en salud dirigidas a la salud infantil y nutricional integral, incluyendo aspectos como la ingesta dietética materna e infantil, aumentar la situación económica del hogar, mejorar el nivel educativo de la madre y equilibrar la distribución de recursos entre diferentes regiones.
\end{abstract}

Palabras clave: Desnutrición; morbilidad; mortalidad; epidemiología; Colombia.
Autor de correspondencia*

${ }^{1 *}$ Enfermera, Especialista en Epidemiología Clínica. Magíster en Enfermería y Candidata a Doctora en Bioética. Docente Universidad de Boyacá. Tunja, Colombia. Correo: mpquemba@ uniboyaca.edu.co. (D) 0000-0001-56466123

2 Médico y cirujano general, Subred Integrada de Servicios de Salud Norte E.S.E. Bogotá, Colombia. Correo: ihulycohe@hotmail.com (iD) 0000-00031152-6203

${ }^{3}$ Médico y Cirujano General, candidata a Magister en Epidemiología, Subred Integrada de Servicios de Salud Norte E.S.E. Bogotá, Colombia. Correo: alejamendoza98@hotmail.com (D) 00000001-5162-0777

${ }^{4}$ Médico y Cirujano General, Subred Integrada de Servicios de Salud Norte E.S.E. Bogotá, Colombia. Correo: benignomo@hotmail.com (iD) 0000-00023483-7023

Recibido: 9 agosto 2021

Aprobado: 29 noviembre 2021

Para citar este artículo

Quemba-Mesa MP, Herrera-Tarapues, Mendoza-Ortiz A, Mendoza-Ortiz B. Comportamiento epidemiológico de la desnutrición en menores de 5 años, Colombia 2016-2019. Rev. cienc. cuidad. 2022; 19(1):71-81. https://doi. org/10.22463/17949831.3186

(C) Universidad Francisco de Paula Santander. Este es un artículo bajo la licencia CC-BY-NC-ND
10,000 inhabitants in contrast to 2019 where this rate increased to 40.5 . The years of life lost due to premature mortality ranged from 1,162 in 2016 to 6,411 in 2019 and the years lived with disability ranged from 588 to 2016 to 2,257 to 2019; disability-adjusted life years ranged

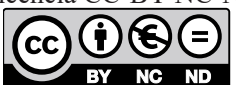




\section{Scientific Journal of Nursing}

from 2,402 in 2016 to 8,668 in 2019. Conclusions: It is necessary to strengthen the interventions from social policies focused on the social determinants in health aimed at child health and comprehensive nutrition, including aspects such as maternal and child dietary intake, improve the economic situation of the home, improve the educational level of the mother and balance the distribution of resources between different regions.

Keywords: Malnutrition; morbidity; mortality; epidemiology; Colombia.

\section{Resumo}

Objetivo: Analisar o comportamento epidemiológico e a prevalência de desnutrição aguda em crianças menores de 5 anos na Colômbia durante os anos de 2016 a 2019. Materiais e métodos: Quantitativo retrospectivo - estudo descritivo; A população e amostra de análise deste estudo é composta por 53.434 relatórios consolidados no Sistema de Informação Integrado de proteção social SISPRO de crianças menores de 5 anos com desnutrição aguda na Colômbia disponíveis destes dos anos de 2016 a 2019. Resultados: Em 2016 existe uma taxa de mortalidade por desnutrição de 0,08 casos por 10.000 habitantes, em comparação com 2019 onde esta taxa aumentou para 0,42; Em 2016, há uma taxa de morbidade por desnutrição aguda de 22,4 casos por 10.000 habitantes, em comparação com 2019 onde essa taxa aumentou para 40,5 . Os anos de vida perdidos por mortalidade prematura variaram de $1.162 \mathrm{em} 2016$ a 6.411 em 2019 e os anos vividos com deficiência variaram de 588 a 2016 a 2.257 a 2019; anos de vida ajustados por incapacidade variaram de $2.402 \mathrm{em} 2016$ a $8.668 \mathrm{em}$ 2019. Conclusões: É necessário fortalecer as intervenções das políticas sociais voltadas aos determinantes sociais em saúde voltadas à saúde infantil e à nutrição integral, incluindo aspectos como o consumo alimentar materno-infantil, melhoria da situação econômica do domicílio, melhoria do nível educacional dos a mãe e equilibrar a distribuição de recursos entre as diferentes regiões.

Palavras chave: Desnutrição, morbidade; mortalidade, epidemiology, Colômbia.

\section{Introducción}

La desnutrición se conceptualiza como una patología de origen social, que expresa directamente situaciones de falta de seguridad alimentaria y nutricional de poblaciones específicas; igualmente afecta de forma directa a la primera infancia, especialmente en menores de 5 años. Esta condición se refleja en alteraciones en la composición del cuerpo y en la funcionalidad de órganos y aspectos psicosociales. Por otra parte, la desnutrición aguda es más frecuente en poblaciones en condiciones de vulnerabilidad por la pobreza, la dificultad en el acceso a servicios de salud, agua y saneamiento básico, lo que les pone en alto riesgo de muerte (1).

Al respecto, la Organización Mundial de la Salud (OMS) y la Organización de las Naciones Unidas para la Alimentación (FAO) muestran que hay en promedio unos 52 millones de niños menores de 5 años que desarrollaron emaciación (malnutrición potencialmente mortal acompañada de delgadez y debilidad extremas) y 17 millones sufren de emaciación grave; además, cerca del $45 \%$ de las muertes en niños menores de 5 años tienen relación con la desnutrición $(2,3)$. En Latinoamérica hasta 53 millones de personas no tienen accesibilidad a alimentos que les proporcionen las necesidades nutricionales requeridas, con mayor incidencia en la población indígena y más en el área rural que en el área urbana (4). Es importante resaltar, que el comportamiento epidemiológico de la desnutrición aguda en Colombia es de $0.9 \%$, con diferencias importantes entre regiones geográficas del país, donde hay dificultades en el acceso a los alimentos por situaciones de conflicto armado, desplazamiento forzado y cambio climático (1).

Estudios a nivel nacional muestran cómo el nivel de riqueza de las comunidades se asocia con la desnutrición crónica en esta población de la primera infancia, además de ser más frecuente en niños de madres que viven en contextos de precarios niveles de autonomía y con dificultades en la accesibilidad al sistema de salud 


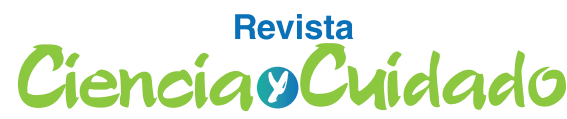

Scientific Journal of Nursing

(5); así, la ubicación espacial de las regiones muestra la coexistencia de la malnutrición por deficiencia y su exceso varía según el área geográfica (6). Lo anterior demuestra la necesidad de que a nivel nacional se aumenten los esfuerzos en la materia y se estructuren modelos de atención integral que incluyan la promoción de la lactancia materna, la introducción oportuna de la alimentación de tipo complementaria y la educación nutricional; así mismo se debe detectar de forma precoz la desnutrición y su control oportuno, entre otras (7); esto desde una visión que incluya los determinantes sociales que sustentan la desnutrición infantil (8).

Por lo anterior, es necesario establecer de forma comparativa cómo las características sociodemográficas varían en las distintas regiones del país y cómo se asocian con la aparición de desnutrición infantil aguda en menores de 5 años; es por eso que el presente estudio propone analizar el comportamiento epidemiológico y la prevalencia de la desnutrición aguda en niños menores de 5 años en Colombia, en el periodo comprendido entre los años 2016 al 2019.

\section{Objetivo}

Analizar el comportamiento epidemiológico y la prevalencia de la desnutrición aguda en niños menores de 5 años en Colombia, durante los años 2016 al 2019.

\section{Materiales y Métodos}

Estudio cuantitativo - descriptivo de tipo retrospectivo. La población y muestra de análisis del presente estudio está conformada por 53.434 reportes consolidados en el Sistema integrado de información de la protección social (SISPRO) de niños y niñas menores de 5 años con desnutrición aguda en Colombia, disponibles durante los años 2016 a 2019 (se aclara que aún no se encuentran consolidados los reportes de 2020), además de los reportes de mortalidad por desnutrición en este mismo rango etario. El muestreo fue por conveniencia, seleccionando a quienes cumplan con los criterios de selección. Los criterios de inclusión fueron los reportes consolidados durante los años 2016 a 2019 de niños y niñas con edades que oscilaban de 0 a 5 años con desnutrición, excluyendo los reportes con datos incompletos sobre la revisión en la base de datos.

De la base de datos SISPRO se tuvieron en cuenta variables como: edad, género, régimen de afiliación en salud, pertenencia étnica, área geográfica, entre otras. El plan de análisis se desarrolló con una fase inicial univariada de acuerdo a la tipología de cada variable; también se calcularon medidas descriptivas como frecuencias, medidas de dispersión y tendencia central. De igual manera, se estimaron los años de vida ajustados por discapacidad (DALYs) individuales, esto como producto de la suma de los años de vida perdidos por mortalidad prematura (YLLs) y los años vividos con discapacidad (YLDs); al respecto se usaron herramientas de la carga global sobre la enfermedad de la OMS (9). Por otra parte, la prevalencia y tasa de morbimortalidad de la población en general por sexo, se aplicó en función de la referencia respecto a las proyecciones de la población comprendida entre 2005 y 2020. A escala nacional por sexo y grupos de edad, se dispuso de los datos proporcionados por el Departamento Administrativo Nacional de Estadísticas (DANE) (10).

Conviene aclarar que este estudio cumplió con los principios éticos para las investigaciones en seres humanos, de acuerdo a la Resolución 8430 de 1993 del Ministerio de Salud de Colombia (11) y de las normas éticas de la Declaración de Helsinki de 1975 (12); por tanto, es un estudio sin riesgo, pues no requiere acceso directo a la población, puesto que se tomó como insumo las bases de datos nacionales existentes en la materia. De igual forma se aplican los principios de responsabilidad y honestidad en el desarrollo del estudio en todas sus fases.

\section{Resultados}

Se estudiaron 53.434 reportes de desnutrición aguda y 501 reportes de mortalidad por desnutrición en niños menores de 5 años en Colombia, durante los años 2016 al 2019.

La morbilidad por desnutrición aguda se presentó con mayor frecuencia en niños de sexo masculino (n:28.854; 53\%), se reportaron mayor número de casos en el año 2019 (n:17.693; 31.8\%), en población de régimen subsidiado ( $\mathrm{n}: 36.974 ; 69.2 \%)$ en residentes en cabecera municipal (n:33.400;62.5\%), pertenecientes a otras etnias (n:38.609; 72.3\%). Respecto a las condiciones de vulnerabilidad, se observó mayor frecuencia en los migrantes (n:1596; 30\%); el estar a cargo del Instituto Colombiano de Bienestar Familiar (ICBF) (n: $840 ; 1.6 \%$ ) y el ser desplazado (n: $737 ; 1.4 \%$ ) (ver tabla $1)$. 
Tabla 1: Desnutrición aguda y mortalidad por desnutrición en niños menores de 5 años, Colombia, 2016- 2019.

\begin{tabular}{|c|c|c|c|c|c|c|c|}
\hline \multicolumn{2}{|r|}{ Variable } & \multicolumn{3}{|c|}{$\begin{array}{c}\text { Morbilidad por desnutrición } \\
\text { aguda }\end{array}$} & \multicolumn{3}{|c|}{ Mortalidad por desnutrición } \\
\hline & & $\begin{array}{l}\text { Mujeres } \\
(\mathrm{n}: 24.580)\end{array}$ & $\begin{array}{l}\text { Hombres } \\
(\mathrm{n}: 28.854)\end{array}$ & $\begin{array}{c}\text { Total } \\
\text { (n:53.434) }\end{array}$ & $\begin{array}{l}\text { Femenino } \\
(\mathrm{n}: 229)\end{array}$ & $\begin{array}{l}\text { Masculino } \\
(\mathrm{n}: 272)\end{array}$ & $\begin{array}{c}\text { Total } \\
(\mathrm{n}: 501)\end{array}$ \\
\hline \multirow[t]{4}{*}{ Año } & 2016 & $\begin{array}{l}4.611 \\
(47 \%)\end{array}$ & $\begin{array}{l}5.103 \\
(53 \%)\end{array}$ & $\begin{array}{c}9.714 \\
(18.2 \%)\end{array}$ & $\begin{array}{c}18 \\
(54.5 \%)\end{array}$ & $\begin{array}{c}33 \\
(6.6 \%)\end{array}$ & $\begin{array}{c}33 \\
(6.6 \%)\end{array}$ \\
\hline & 2017 & $\begin{array}{l}4.901 \\
(46 \%)\end{array}$ & $\begin{array}{l}5.740 \\
(54 \%)\end{array}$ & $\begin{array}{c}10.641 \\
(19.9 \%)\end{array}$ & $\begin{array}{c}66 \\
(52.8 \%)\end{array}$ & $\begin{array}{c}125 \\
(25.0 \%)\end{array}$ & $\begin{array}{c}125 \\
(25.0 \%)\end{array}$ \\
\hline & 2018 & $\begin{array}{l}7.106 \\
(46 \%)\end{array}$ & $\begin{array}{l}8.280 \\
(54 \%)\end{array}$ & $\begin{array}{c}15.386 \\
(28.8 \%)\end{array}$ & $\begin{array}{c}85 \\
(52 . \%)\end{array}$ & $\begin{array}{c}161 \\
(32.1 \%)\end{array}$ & $\begin{array}{c}161 \\
(32.1 \%)\end{array}$ \\
\hline & 2019 & $\begin{array}{l}7.962 \\
(45 \%)\end{array}$ & $\begin{array}{l}9.731 \\
(55 \%)\end{array}$ & $\begin{array}{l}17.693 \\
(31.8 \%)\end{array}$ & $\begin{array}{c}103 \\
(56.6 \%)\end{array}$ & $\begin{array}{c}182 \\
(36.3 \%)\end{array}$ & $\begin{array}{c}182 \\
(36.3 \%)\end{array}$ \\
\hline \multirow[t]{5}{*}{$\begin{array}{l}\text { Tipo de rég- } \\
\text { imen }\end{array}$} & Subsidiado & $\begin{array}{c}17.268 \\
(46.7 \%)\end{array}$ & $\begin{array}{l}19.706 \\
(53.3 \%)\end{array}$ & $\begin{array}{c}36.974 \\
(69.2 \%)\end{array}$ & $\begin{array}{c}224 \\
(54.4 \%)\end{array}$ & $\begin{array}{c}412 \\
(82.2 \%)\end{array}$ & $\begin{array}{c}412 \\
(82.2 \%)\end{array}$ \\
\hline & Contributivo & $\begin{array}{c}4.678 \\
(42.8 \%)\end{array}$ & $\begin{array}{c}6.261 \\
(57.2 \%)\end{array}$ & $\begin{array}{l}10.939 \\
(20.5 \%)\end{array}$ & $\begin{array}{c}8 \\
(50.0 \%)\end{array}$ & $\begin{array}{c}16 \\
(3.2 \%)\end{array}$ & $\begin{array}{c}16 \\
(3.2 \%)\end{array}$ \\
\hline & Especial & $\begin{array}{c}119 \\
(46.5 \%)\end{array}$ & $\begin{array}{c}137 \\
(53.5 \%)\end{array}$ & $\begin{array}{c}256 \\
(0.5 \%)\end{array}$ & $\begin{array}{c}0 \\
(0 \%)\end{array}$ & $\begin{array}{c}1 \\
(0.2 \%)\end{array}$ & $\begin{array}{c}1 \\
(0.2 \%)\end{array}$ \\
\hline & Excepción & $\begin{array}{c}91 \\
(46.9 \%)\end{array}$ & $\begin{array}{c}103 \\
(53.1 \%)\end{array}$ & $\begin{array}{c}194 \\
(0.4 \%)\end{array}$ & $\begin{array}{c}0 \\
(0 \%)\end{array}$ & $\begin{array}{c}1 \\
(0.2 \%)\end{array}$ & $\begin{array}{c}1 \\
(0.2 \%)\end{array}$ \\
\hline & No afiliado / no definido & $\begin{array}{c}2.424 \\
(47.8 \%)\end{array}$ & $\begin{array}{c}2.647 \\
(52.2 \%)\end{array}$ & $\begin{array}{l}5.071 \\
(9.5 \%)\end{array}$ & $\begin{array}{c}40 \\
(56.3 \%)\end{array}$ & $\begin{array}{c}71 \\
(14.2 \%)\end{array}$ & $\begin{array}{c}71 \\
(14.2 \%)\end{array}$ \\
\hline \multirow[t]{3}{*}{ Área } & Cabecera & $\begin{array}{c}15.165 \\
(45.4 \%)\end{array}$ & $\begin{array}{c}18.235 \\
(54.6 \%)\end{array}$ & $\begin{array}{l}33.400 \\
(62.5 \%)\end{array}$ & $\begin{array}{c}127 \\
(55.5 \%)\end{array}$ & $\begin{array}{c}229 \\
(45.7 \%)\end{array}$ & $\begin{array}{c}229 \\
(45.7 \%)\end{array}$ \\
\hline & Centro poblado & $\begin{array}{c}2.678 \\
(48.0 \%)\end{array}$ & $\begin{array}{c}2.905 \\
(52.0 \%)\end{array}$ & $\begin{array}{c}5.583 \\
(38.6 \%)\end{array}$ & $\begin{array}{c}39 \\
(61.9 \%)\end{array}$ & $\begin{array}{c}63 \\
(12.6 \%)\end{array}$ & $\begin{array}{c}63 \\
(12.6 \%)\end{array}$ \\
\hline & Área rural dispersa & $\begin{array}{c}6.737 \\
(46.6 \%)\end{array}$ & $\begin{array}{c}7.714 \\
(53.4 \%)\end{array}$ & $\begin{array}{c}14.451 \\
(27.0 \%)\end{array}$ & $\begin{array}{c}106 \\
(50.7 \%)\end{array}$ & $\begin{array}{c}209 \\
(41.7 \%)\end{array}$ & $\begin{array}{c}209 \\
(41.7 \%)\end{array}$ \\
\hline \multirow[t]{6}{*}{$\begin{array}{l}\text { Pertenencia } \\
\quad \text { étnica }\end{array}$} & Indígena & $\begin{array}{c}5.147 \\
(46.4 \%)\end{array}$ & $\begin{array}{c}5.936 \\
(53.6 \%)\end{array}$ & $\begin{array}{c}11.083 \\
(20.7 \%)\end{array}$ & $\begin{array}{c}127 \\
(54.3 \%)\end{array}$ & $\begin{array}{c}234 \\
(46.7 \%)\end{array}$ & $\begin{array}{c}234 \\
(46.7 \%)\end{array}$ \\
\hline & Gitano & $\begin{array}{c}96 \\
(48.2 \%)\end{array}$ & $\begin{array}{c}103 \\
(51.8 \%)\end{array}$ & $\begin{array}{c}199 \\
(0.4 \%)\end{array}$ & $\begin{array}{c}0 \\
(0 \%)\end{array}$ & $\begin{array}{c}0 \\
(0 \%)\end{array}$ & $\begin{array}{c}0 \\
(0 \%)\end{array}$ \\
\hline & $\begin{array}{l}\text { Raizal (San Andrés y } \\
\text { Providencia) }\end{array}$ & $\begin{array}{c}55 \\
(57.9 \%)\end{array}$ & $\begin{array}{c}40 \\
(42.1 \%)\end{array}$ & $\begin{array}{c}95 \\
(0.2 \%)\end{array}$ & $\begin{array}{c}0 \\
(0 \%)\end{array}$ & $\begin{array}{c}0 \\
(0 \%)\end{array}$ & $\begin{array}{c}0 \\
(0 \%)\end{array}$ \\
\hline & Palanquero de San Basilio & $\begin{array}{c}30 \\
(65.2 \%)\end{array}$ & $\begin{array}{c}16 \\
(34.8 \%)\end{array}$ & $\begin{array}{c}46 \\
(0.1 \%)\end{array}$ & $\begin{array}{c}0 \\
(0 \%)\end{array}$ & $\begin{array}{c}0 \\
(0 \%)\end{array}$ & $\begin{array}{c}0 \\
(0 \%)\end{array}$ \\
\hline & $\begin{array}{c}\text { Negro, mulato, afrocolom- } \\
\text { biano }\end{array}$ & $\begin{array}{c}1.608 \\
(47.3 \%)\end{array}$ & $\begin{array}{c}1.794 \\
(52.7 \%)\end{array}$ & $\begin{array}{l}3.402 \\
(6.4 \%)\end{array}$ & $\begin{array}{c}14 \\
(43.8 \%)\end{array}$ & $\begin{array}{c}32 \\
(6.4 \%)\end{array}$ & $\begin{array}{c}32 \\
(6.4 \%)\end{array}$ \\
\hline & Otras etnias & $\begin{array}{c}17.644 \\
(45.7 \%)\end{array}$ & $\begin{array}{c}20.965 \\
(54.3 \%)\end{array}$ & $\begin{array}{c}38.609 \\
(72.3 \%)\end{array}$ & $\begin{array}{c}132 \\
(55.9 \%)\end{array}$ & $\begin{array}{c}236 \\
(47.1 \%)\end{array}$ & $\begin{array}{c}236 \\
(47.1 \%)\end{array}$ \\
\hline
\end{tabular}




\section{Scientific Journal of Nursing}

\begin{tabular}{cccccccc}
$\begin{array}{c}\text { Condiciones } \\
\text { de vulnerab- } \\
\text { ilidad }\end{array}$ & $\begin{array}{c}\text { Víctima de violencia } \\
\text { armada }\end{array}$ & 68 & 88 & 156 & 3 & 5 & 5 \\
& A cargo del ICBF & $46.3 \%)$ & $(56.4 \%)$ & $(0.3 \%)$ & $(60.0 \%)$ & $(9.3 \%)$ & $(9.3 \%)$ \\
& & 411 & 840 & 9 & 18 & 18 \\
& Migrantes & 714 & 882 & 1596 & 11 & 18 & 18 \\
& & $(44.7 \%)$ & $(55.3 \%)$ & $(3.0 \%)$ & $(61.1 \%)$ & $(33.3 \%)$ & $(33.3 \%)$ \\
& Población indigente & 72 & 109 & 181 & 1 & 3 & 3 \\
& & $(39.8 \%)$ & $(60.2 \%)$ & $(0.3 \%)$ & $(33.3 \%)$ & $(5.6 \%)$ & $(5.6 \%)$ \\
& & 91 & 124 & 215 & 3 & 3 & 3 \\
& Discapacidad & $(42.3 \%)$ & $(57.7 \%)$ & $(0.4 \%)$ & $(100 \%)$ & $(5.6 \%)$ & $(5.6 \%)$ \\
& & 337 & 400 & 737 & 4 & 5 & 5 \\
& Desplazados & $(45.7 \%)$ & $(54.3 \%)$ & $(1.4 \%)$ & $(80 \%)$ & $(9.3 \%)$ & $(9.3 \%)$ \\
& & 7 & 17 & 24 & 1 & 1 & 1 \\
& Desmovilizados & $(29.2 \%)$ & $(70.8 \%)$ & $(0.04 \%)$ & $(100 \%)$ & $(1.9 \%)$ & $(1.9 \%)$ \\
& & 2 & 12 & 14 & 1 & 1 & 1 \\
& Carcelaria & $(14.3 \%)$ & $(85.7 \%)$ & $(0.03 \%)$ & $(100 \%)$ & $(1.9 \%)$ & $(1.9 \%)$ \\
\hline
\end{tabular}

Fuente: Elaborada por autores

La mortalidad por desnutrición en esta población se presentó con mayor frecuencia en niños de sexo masculino (n:272; 54\%), reportándose el mayor número de casos en el año 2019 (n:182; 36.3\%) y en población de régimen subsidiado ( $\mathrm{n}: 412 ; 82.2 \%$ ); residentes en cabecera municipal (n:229; 45.7\%); pertenecientes a otras etnias (n:236; 47.1\%). Desde otro punto de vista, las condiciones de vulnerabilidad de mayor frecuencia son: ser migrante (n:18; 33.3\%), estar a cargo del ICBF (n:18; 33.3\%), ser desplazado (n:5; 9.3\%) y víctima de violencia armada (n:5; 9.3\%) (ver tabla 1$)$.

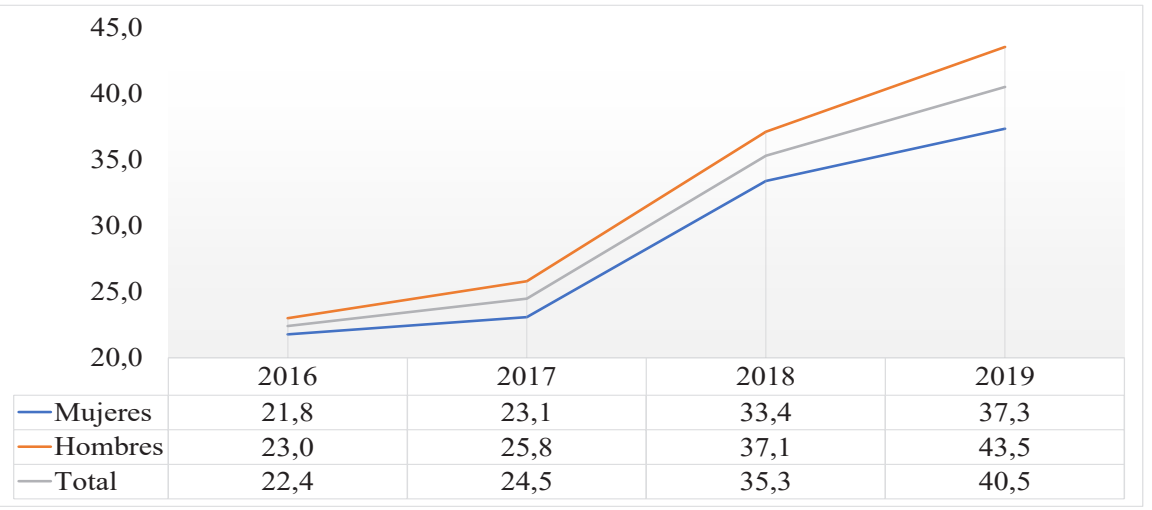

Figura 1: Tasa por 10,000 habitantes de desnutrición aguda en niños menores de 5 años en Colombia, 2016 al 2019.

Fuente: Elaborada por autores.

Tanto la prevalencia como la tasa de morbilidad y mortalidad relacionada con la desnutrición en niños menores de 5 años presenta una tendencia a aumentar si se comparan los años 2016 al 2019 para estos indicadores, así: en el 2016 se presenta una tasa de mortalidad por desnutrición de 0.08 casos por cada 10.000 habitantes, en contraste con el 2019, en donde esta tasa aumenta a 0,42 casos por cada 10.000 habitantes (ver tabla 2). Por otro lado, en el 2016 hay una tasa de morbilidad por desnutrición aguda de 22,4 casos por cada 10.000 habitantes, en contraste con el 2019 donde ésta tasa aumentó a 40,5 casos por cada 10.000 habitantes (ver Figura 1; ver tabla 2). 
Scientific Journal of Nursing

Tabla 2. Carga de la desnutrición en niños menores de 5 años en Colombia, 2016 al 2019

\begin{tabular}{|c|c|c|c|c|c|c|c|c|c|c|c|}
\hline & & \multicolumn{2}{|c|}{ Mortalidad } & \multicolumn{2}{|c|}{ Morbilidad } & \multicolumn{2}{|c|}{ YLLs } & \multicolumn{2}{|c|}{ YLDs } & \multicolumn{2}{|c|}{$\begin{array}{c}\text { Total, DALYS } \\
\text { YLL+YLD }\end{array}$} \\
\hline & & $\begin{array}{l}\text { Prevalen- } \\
\text { cia* }\end{array}$ & $\begin{array}{l}\text { Tasa por } \\
10.000^{*}\end{array}$ & $\begin{array}{l}\text { Prevalen- } \\
\text { cia* }\end{array}$ & $\begin{array}{l}\text { Tasa por } \\
10.000^{*}\end{array}$ & YLLs* & $\begin{array}{l}\text { YLLs } \\
\text { por } \\
1,000^{*}\end{array}$ & YLDs* & $\begin{array}{l}\text { YLDs } \\
\text { por } \\
1.000^{*}\end{array}$ & DALYs* & $\begin{array}{l}\text { DALYs } \\
\text { por } \\
1.000^{*}\end{array}$ \\
\hline \multirow[t]{3}{*}{2016} & Mujeres & $0,0007 \%$ & 0,07 & $0,218 \%$ & 21,8 & 529 & 0,3 & 588 & 0,3 & 1.284 & 0,6 \\
\hline & Hombres & $0,0008 \%$ & 0,08 & $0,230 \%$ & 23,0 & 633 & 0,3 & 651 & 0,3 & 1.118 & 0,5 \\
\hline & Total & $0,0008 \%$ & 0,08 & $0,224 \%$ & 22,4 & 1162 & -- & 1239 & -- & 2.402 & 0,6 \\
\hline \multirow[t]{3}{*}{2017} & Mujeres & $0,0028 \%$ & 0,28 & $0,231 \%$ & 23,1 & 2.082 & 1,0 & 625 & 0,3 & 2.707 & 1,3 \\
\hline & Hombres & $0,0030 \%$ & 0,30 & $0,258 \%$ & 25,8 & 2.321 & 1,0 & 732 & 0,3 & 3.053 & 1,4 \\
\hline & Total & $0,0029 \%$ & 0,29 & $0,245 \%$ & 24,5 & 4.403 & -- & 1357 & -- & 5.760 & 1,3 \\
\hline \multirow[t]{3}{*}{2018} & Mujeres & $0,0036 \%$ & 0,36 & $0,334 \%$ & 33,4 & 2.682 & 1,3 & 906 & 0,4 & 3.589 & 1,7 \\
\hline & Hombres & $0,0038 \%$ & 0,38 & $0,371 \%$ & 37,1 & 2.990 & 1,3 & 1056 & 0,5 & 4.046 & 1,8 \\
\hline & Total & $0,0037 \%$ & 0,37 & $0,353 \%$ & 35,3 & 5.672 & -- & 1.962 & -- & 7.634 & 1,8 \\
\hline \multirow[t]{3}{*}{2019} & Mujeres & $0,0037 \%$ & 0,37 & $0,373 \%$ & 37,3 & 2.788 & 1,3 & 1.016 & 0,5 & 3.804 & 1,8 \\
\hline & Hombres & $0,0046 \%$ & 0,46 & $0,435 \%$ & 43,5 & 3.623 & 1,6 & 1.241 & 0,6 & 4.864 & 2,2 \\
\hline & Total & $0,0042 \%$ & 0,42 & $0,405 \%$ & 40,5 & 6.411 & -- & 2.257 & -- & 8.668 & 2,0 \\
\hline
\end{tabular}

(*) Para el cálculo de estos indicadores se tomó como población de referencia las proyecciones de población 2005-2020, a escala nacional por sexo y grupos de edad, del Departamento Administrativo Nacional de Estadísticas (DANE) (10).

Fuente: Elaborada por autores

Los departamentos con mayor número de reportes de morbilidad por desnutrición en esta población son: Cundinamarca $(7.588 ; 14,2 \%)$, Bogotá, D.C. $(6.159$; $11,5 \%)$ y la Guajira $(4.750 ; 8,9 \%)$; contrariamente, los departamentos con mayor número de reportes de mortalidad por desnutrición son la Guajira $(84 ; 16.8 \%)$, Cesar (32; 6.4\%) y Chocó $(31 ; 6.2 \%)$ (ver figura 2$)$.

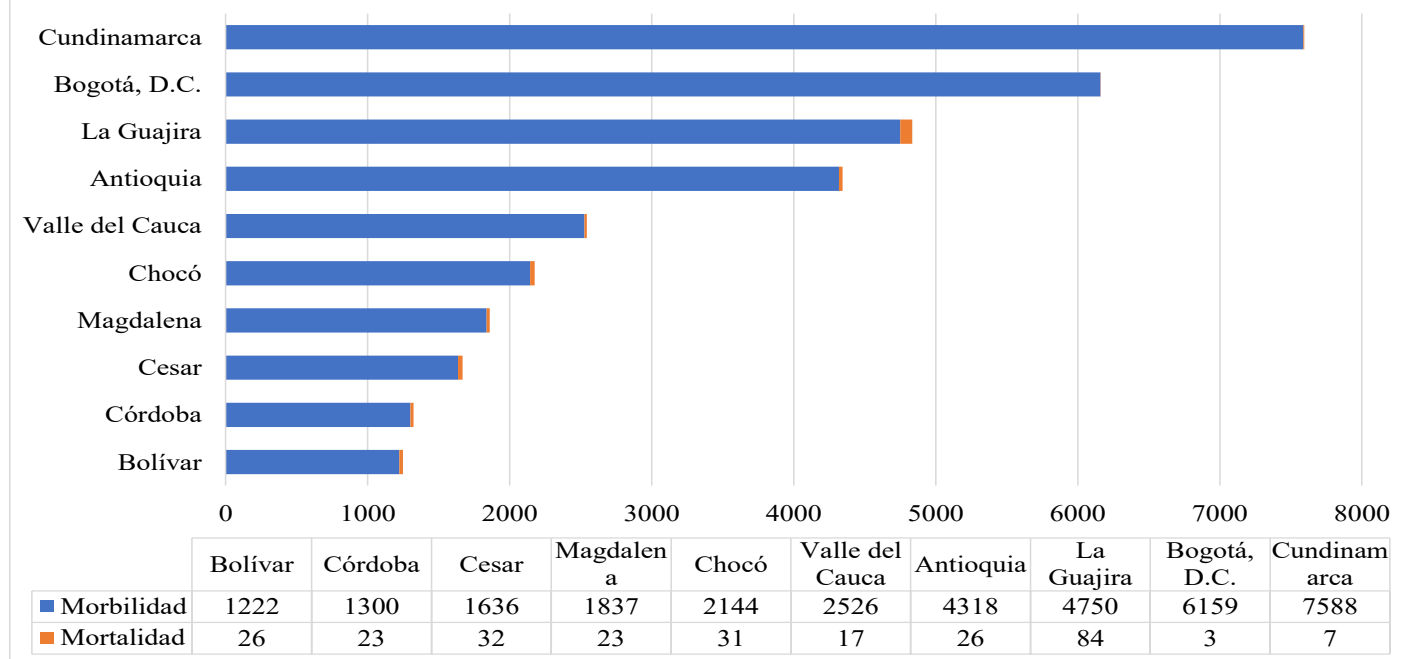

Figura 2: Departamentos con mayor número de reportes de morbilidad y mortalidad por desnutrición, en niños menores de 5 años en Colombia, 2016 al 2019

Fuente: Elaborada por autores. 
En cuanto a los indicadores de carga de desnutrición en menores de 5 años en Colombia y los años de vida perdidos por mortalidad prematura (YLLs) oscilaron entre 1.162 en el 2016 a 6.411 en el 2019; los años vividos con discapacidad (YLDs) oscilaron entre 588 en
2016 a 2.257 en 2019 (ver tabla 2). En cambio, los años de vida ajustados por discapacidad (DALYs) oscilaron entre 2.402 en 2016 a 8.668 en el 2019 (ver tabla 2 y figura 3).

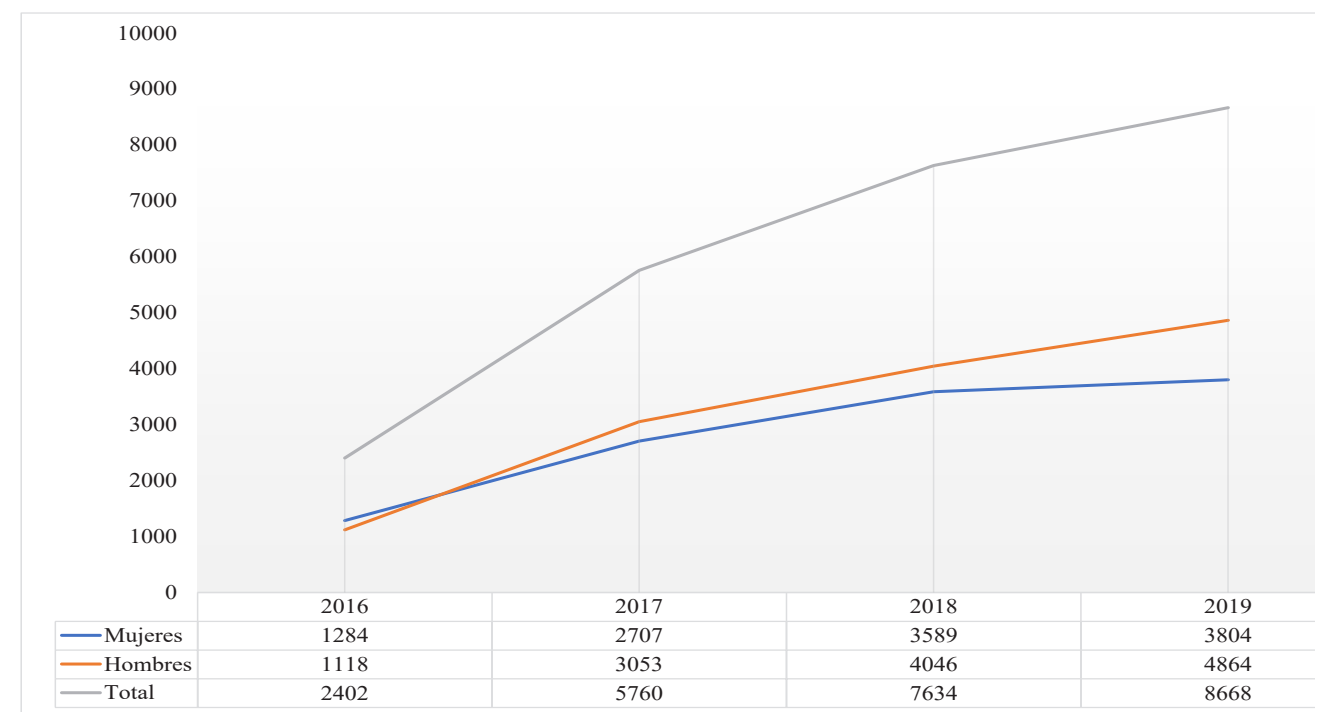

Figura 3: Años de vida perdidos ajustados por discapacidad (AVAD o DALY) y por desnutrición en niños menores de 5 años en Colombia, 2016 al 2019

Fuente: Elaborada por autores

\section{Discusión}

El presente estudio mostró el comportamiento y las características de los reportes de desnutrición aguda y mortalidad por desnutrición en niños menores de 5 años en Colombia durante los años de 2016 a 2019, con una importante frecuencia de características relacionadas con determinantes sociales en salud desfavorables, presentes en estos casos. Se observa con mayor frecuencia en la población de régimen subsidiado, el área de residencia, la pertenencia étnica y el hacer parte de poblaciones con condiciones especiales de vulnerabilidad, como ser migrante, víctima de la violencia, desplazado y estar a cargo del ICBF, entre otros. En este contexto, estudios con esta metodología, permiten caracterizar este fenómeno y calcular indicadores relacionados con la carga de la enfermedad que permiten identificar el impacto en la salud pública sobre diferentes problemas de salud y factores de riesgo (13).

En Colombia, si bien se han desarrollado estrategias transectoriales para mitigar esta temática, como es el caso de la Política de Estado de Cero a Siempre, que vela desde el año 2016 por el Desarrollo Integral de la Primera Infancia incluyendo temas sensibles como su protección, su educación y por supuesto su salud y nutrición (14), sin embargo, al confrontar los resultados de este comportamiento epidemiológico, se evidencia un aumento sostenido desde el 2016 al 2019, tanto en el reporte de casos de morbilidad como de mortalidad por desnutrición en menores de 5 años. Resultados que cuestionan el impacto real de este tipo de esfuerzos gubernamentales, sin dejar de lado el fortalecimiento que se ha dado a los sistemas nacionales de identificación y reporte de este tipo de eventos de interés en salud pública. En este contexto, sigue siendo desafortunado el número de niños y niñas que sufren este flagelo a nivel nacional.

En países como Etiopía la prevalencia de desnutrición aguda es de 5,4\% (15) y en Nepal de 4.14\% (16), 10 veces mayor que lo encontrado en Colombia en este estudio (0,405\% para el 2019). No obstante, es común la presencia de problemáticas relacionadas con el nivel socioeconómico bajo, el no brindar educación sanitaria a las madres sobre higiene personal y ambiental y la 
eliminación inadecuada de desechos; estos son aspectos esenciales que se deben tener en cuenta desde un enfoque multisectorial para minimizar el problema de la desnutrición $(15,16)$; además hay que fortalecer la atención en salud materna, la inmunización completa y la lactancia materna temprana (17). Se debe mencionar que en investigaciones realizadas en países como $\mathrm{Pa}-$ kistán, se muestra el impacto de la desnutrición en el retraso sobre el crecimiento de los niños (18), lo cual motiva a que se amplíen los estudios a nivel nacional, de tal forma que permitan establecer las profundas secuelas que este flagelo está ocasionando en esta población y sobre todo dar luces sobre cómo mitigarlas, dado que la desnutrición puede influir negativamente en la salud de las generaciones venideras y por tanto en el progreso de las naciones (19).

Por otra parte, el pertenecer a grupos sociales que históricamente son caracterizados como vulnerables por la estigmatización que las sociedades generan hacia estos, es un factor que incide en el desarrollo de la desnutrición infantil. Hay que mencionar que en el presente estudio se identificó que un importante número de niños pertenecían a grupos étnicos y hacían parte de poblaciones con condiciones especiales de vulnerabilidad, como el ser migrantes, víctimas de la violencia, desplazados, estar a cargo del ICBF, entre otros. Al respecto, en Serbia se estableció que los niños que pertenecen a las étnicas romaníes, tenían tres veces más probabilidades de presentar retraso en el crecimiento y / o un retraso en el crecimiento grave frente a los niños no romaníes del quintil de riqueza más baja (20).

En relación al desplazamiento, en 2015 de los más de 1 millón de migrantes que solicitaron asilo en Europa, casi un tercio eran niños y 90.000 no estaban acompañados; razón por la cual estos niños se encuentran entre los más vulnerables, pues su salud depende de su estado de salud antes del viaje, las condiciones durante su viaje y en su destino, así como de la salud física y mental de sus cuidadores (21). Conviene subrayar que el tiempo de desplazamiento de los migrantes aumenta el riesgo de desnutrición, así como las condiciones de vida y los problemas de salud en esta población, implicando necesariamente una mejor protección de los migrantes, refugiados y solicitantes de asilo (22).

Se sabe que la población víctima de desplazamiento vive en inseguridad alimentaria y con una mala salud poblacional (23); de ahí, que en las poblaciones de refugiados el riesgo de desnutrición y otras complicaciones en salud esté más latente (24).

En general, todas las condiciones sociales que generen inseguridad alimentaria en los hogares están asociadas con los problemas de comportamiento, los académicos y emocionales de los niños desde la infancia hasta la adolescencia (25). Lo planteado anteriormente, obliga a invertir en intervenciones específicas de nutrición, para evitar la desnutrición materna e infantil y las deficiencias de micronutrientes en los segmentos pobres de la población con mayor riesgo, lo que marcaría una gran diferencia. Por tanto, se deben incluir políticas sociales beneficiosas para la promoción de la nutrición infantil, que incorporen aspectos como la ingesta dietética materna e infantil, mejorar la situación económica del hogar, usar alimentos terapéuticos para la rehabilitación nutricional domiciliaria, mejorar el nivel educativo de la madre y equilibrar el desarrollo económico y la distribución de recursos entre diferentes regiones $(26,27,28)$. Estas acciones en los primeros años de la infancia pueden ser eficaces para reducir la desigualdad en la salud nutricional en las zonas rurales (29), siempre haciendo énfasis en la educación basada en la comunidad, en las intervenciones nutricionales específicas (30) y en la trasformación de los determinantes sociales que afectan la salud propia, en función de los contextos geográficos de las distintas regiones $(31,32)$.

En definitiva, se identifican como limitaciones el no poder acceder a más información individualizada y a las percepciones de los cuidadores familiares, además de las condiciones de vida específicas del contexto en los casos reportados. Se sugiere la realización de estudios centrados en las condiciones de vida, las percepciones e intervenciones en torno a este fenómeno.

\section{Conclusiones}

- Los reportes de desnutrición aguda y mortalidad por desnutrición en niños menores de 5 años en Colombia durante los años 2016 al 2019 prevalecen en la región. Así, su comportamiento epidemiológico se describe al contar con una importante frecuencia de características relacionadas con determinantes sociales en salud que son desfavorables en estos casos, afectando principalmente a personas de régimen subsidiado, la pertenencia étnica y el hacer parte de poblaciones con condiciones especiales de vulnerabilidad como: ser migrante, víctima de la 
violencia, desplazado, estar a cargo del ICBF, entre otros.

- Este evento de interés en salud pública, genera una importante carga sobre la enfermedad al sistema de salud, así como a las dinámicas del país, esto en función del número de años de vida perdidos por mortalidad prematura, de años vividos con discapacidad y de años de vida perdidos ajustados por discapacidad.

- En consecuencia, es necesario el fortalecimiento de las intervenciones desde las políticas sociales enfocadas en los determinantes sociales en salud, dirigidas especialmente a la salud infantil y nutricional integral; de ahí que se deban incluir aspectos relacionados con: la ingesta dietética materna e infantil, mejorar la situación económica del hogar, mejorar el nivel educativo de la madre y equilibrar el desarrollo económico y la distribución de recursos entre diferentes regiones.

\section{Conflicto de intereses}

Los autores declaran no tener ningún conflicto de intereses.

\section{Referencias bibliográficas}

1. Ministerio de Salud y Protección Social y Fondo de las Naciones Unidas para la Infancia (UNICEF). Lineamiento para el manejo integrado de la desnutrición aguda moderada y severa en niños y niñas de 0 a 59 meses de edad. [Internet]. [Consultado el 28 de septiembre de 2021]. Disponible en: https://www.minsalud.gov.co/ sites/rid/Lists/BibliotecaDigital/RIDE/VS/PP/lineamiento-desnutricion-aguda-minsalud-unicef-final.pdf

2. Organización Mundial de la Salud (OMS). Malnutrición [Internet]. 09 junio de 2021 [consultado el 02 de septiembre de 2021]. Disponible en: https://www.who.int/es/news-room/fact-sheets/detail/malnutrition

3. Organización de las Naciones Unidas para la Alimentación (FAO). El estado de la seguridad alimentaria y la nutrición en el mundo. Fomentando la resiliencia climática en aras de la seguridad alimentaria y la nutrición. FAO, Roma. [internet] 2018. [Consultado el 02 de septiembre de 2021]. Disponible en: http://www.fao.org/3/ i9553es/i9553es.pdf

4. Ministerio de Salud, Instituto Nacional de Salud. Desnutrición aguda moderada y severa en menores de cinco años. Protocolo de vigilancia en salud pública versión 3 [Internet]. 29 dic de 2020 [consultado el 02 de septiembre de 2021]. Disponible en: https://www.ins.gov.co/buscador-eventos/Lineamientos/Pro Desnutricion $\% 20 \mathrm{en} \% 20$ menores $\% 20 \mathrm{de} \% 205 \% 20 \mathrm{a} \% \mathrm{C} 3 \% \mathrm{~B} 1$ os.pdf

5. Osorio AM, Romero GA, Bonilla H, Aguado LF. Socioeconomic context of the community and chronic child malnutrition in Colombia. Revista de Saúde Pública [online]. 2018; [Accedido 28 Setiembre 2021]: 52(73). Disponible en: https://doi.org/10.11606/S1518-8787.2018052000394

6. Bernal J, Agudelo-Martínez A, Roldan-Jaramillo P. Representación geográfica de la malnutrición en niños y adolescentes de Medellín, Colombia. Rev Esp Nutr Hum Diet [Internet]. 2020 Jun [citado 2021 Sep 28]; 24(2):111-119. Disponible en: https://dx.doi.org/10.14306/renhyd.24.2.945

7. Álvarez MC, López A, Estrada A. Estado nutricional de niños de Antioquia, Colombia, según dos sistemas de referencia. Rev Panam Salud Publica/Pan Am J Public Health [Internet]. 2009 [citado 2021 Sep 28]; 25(3); 196-203. Disponible en: https://scielosp.org/article/rpsp/2009.v25n3/196-203/

8. Vargas M, Hernández E. Los determinantes sociales de la desnutrición infantil en Colombia vistos desde la medicina familiar [The social determinants of child malnutrition in Colombia from a family medicine perspective]. Medwave. 2020;20(2):e7839. https://doi.org/10.5867/medwave.2020.02.7839

9. Organización Mundial de la Salud (OMS). Health statistics and information systems. WHO [internet] 2017 [acceso septiembre 05 de 2019]. Disponible en: https://apps.who.int/iris/handle/10665/143368 
Scientific Journal of Nursing

10. Departamento Administrativo Nacional de Estadísticas (DANE). Proyecciones de población calculadas con base en los resultados del Censo Nacional de Población y Vivienda - CNPV- 2018. 2021 [acceso septiembre 05 de 2019]. Disponible en: https://www.dane.gov.co/index.php/estadisticas-por-tema/demografia-y-poblacion/proyecciones-de-poblacion

11. Ministerio de Salud de Colombia de la República de Colombia. Resolución 8430 de 1993 Por la cual se establecen las normas científicas, técnicas y administrativas para la investigación en salud. [acceso septiembre 05 de 2021]. Disponible: https:/www.minsalud.gov.co/sites/rid/Lists/BibliotecaDigital/RIDE/DE/DIJ/RESOLUCION-8430-DE-1993.PDF

12. Asociación Médica Mundial. Declaración de Helsinki. [acceso septiembre 05 de 2021]. Disponible en: https:// www.wma.net/es/policies-post/declaracion-de-helsinki-de-la-amm-principios-eticos-para-las-investigaciones-medicas-en-seres-humanos/

13. Haagsma JA, Polinder S, Stein CE, Havelaar AH. Systematic review of foodborne burden of disease studies: quality assessment of data and methodology. Int J Food Microbiol. 2013 Aug 16;166(1):34-47. https://doi. org/10.1016/j.ijfoodmicro.2013.05.029

14. Gobierno de Colombia. De Cero a Siempre. [acceso septiembre 05 de 2021]. Disponible en: http://www.deceroasiempre.gov.co/QuienesSomos/Paginas/QuienesSomos.aspx

15. Wasihun AG, Dejene TA, Teferi M, Marugán J, Negash L, Yemane D, et al. Risk factors for diarrhoea and malnutrition among children under the age of 5 years in the Tigray Region of Northern Ethiopia. PLoS One. 2018 Nov 26;13(11):e0207743. https://doi.org/10.1371/journal.pone.0207743

16. Pravana NK, Piryani S, Chaurasiya SP, Kawan R, Thapa RK, Shrestha S. Determinants of severe acute malnutrition among children under 5 years of age in Nepal: a community-based case-control study. BMJ Open. 2017 Aug 28;7(8):e017084. https://doi.org/10.1136/bmjopen-2017-017084

17. Pratim Roy M. Malnutrition in children and its determinants: a study from east India. Trop Doct. 2019 Apr;49(2):113-117. https://doi.org/10.1177/0049475518824825

18. Ali A. Current Status of Malnutrition and Stunting in Pakistani Children: What Needs to Be Done? J Am Coll Nutr. 2021 Feb;40(2):180-192. https://doi.org/10.1080/07315724.2020.1750504

19. Das JK, Lassi ZS, Hoodbhoy Z, Salam RA. Nutrition for the Next Generation: Older Children and Adolescents. Ann Nutr Metab. 2018;72 Suppl 3:56-64. https://doi.org/10.1159/000487385

20. Brcanski J, Jović-Vraneš A, Marinković J, Favre D. Social determinants of malnutrition among Serbian children aged $<5$ years: ethnic and regional disparities. Int J Public Health. 2014 Oct;59(5):697-706. https://doi. org/10.1007/s00038-014-0591-5

21. ISSOP Migration Working Group. ISSOP position statement on migrant child health. Child Care Health Dev. 2018; 44(1):161-170. https://doi.org/10.1111/cch.12485

22. Kuehne A, van Boetzelaer E, Alfani P, et al. Health of migrants, refugees and asylum seekers in detention in Tripoli, Libya, 2018-2019: Retrospective analysis of routine medical programme data. PLoS One. 2021;16(6):e0252460. https://doi.org/10.1371/journal.pone.0252460

23. Lindvall K, Kinsman J, Abraha A, Dalmar A, Abdullahi MF, Godefay H, et al. Health Status and Health Care Needs of Drought-Related Migrants in the Horn of Africa-A Qualitative Investigation. Int J Environ Res Public Health. 2020 Aug 14;17(16):5917. https://doi.org/10.3390/ijerph17165917

24. Sap S, Wandji-Nzebia Y, Dohvoma-Andin V, Hagbe PY, Mbono R, Fotang-Tangmoh I, et al. A hidden face of migration: Diabetic ketoacidosis in a severely malnourished refugee. Clin Case Rep. 2019 Nov 6;7(12):24252428. https://doi.org/10.1002/ccr3.2508

25. Shankar P, Chung R, Frank DA. Association of Food Insecurity with Children's Behavioral, Emotional, and Academic Outcomes: A Systematic Review. J Dev Behav Pediatr. 2017 Feb/Mar;38(2):135-150. https://doi. 
Scientific Journal of Nursing

org/10.1097/DBP.0000000000000383

26. Man SL, Guo Y. Research on the social determinants of malnutrition among children under the age of 5 in China. Journal of Peking University (Health Sciences). 2016 Jun 18;48(3):418-23

27. Zaragoza-Cortes J, Trejo-Osti LE, Ocampo-Torres M, Maldonado-Vargas L, Ortiz-Gress AA. Poor breastfeeding, complementary feeding and dietary diversity in children and their relationship with stunting in rural communities. Nutr Hosp. 2018 Feb 27;35(2):271-278. https://doi.org/10.20960/nh.1352. PMID: 29756958

28. Schoonees A, Lombard MJ, Musekiwa A, Nel E, Volmink J. Ready-to-use therapeutic food (RUTF) for homebased nutritional rehabilitation of severe acute malnutrition in children from six months to five years of age. Cochrane Database Syst Rev. 2019 May 15;5(5):CD009000. https://doi.org/10.1002/14651858.CD009000

29. Zhang N, Bécares L, Chandola T. Patterns and Determinants of Double-Burden of Malnutrition among Rural Children: Evidence from China. PLoS One. 2016 Jul 8;11(7):e0158119. https://doi.org/10.1371/journal. pone.0158119

30. Khan S, Zaheer S, Safdar NF. Determinants of stunting, underweight and wasting among children $<5$ years of age: evidence from 2012-2013 Pakistan demographic and health survey. BMC Public Health. 2019 Apr 1;19(1):358. https://doi.org/10.1186/s12889-019-6688-2

31. Beiersmann C, Bermejo Lorenzo J, Bountogo M, Tiendrébeogo J, Gabrysch S, Yé M, Jahn A, Müller O. Malnutrition determinants in young children from Burkina Faso. J Trop Pediatr. 2013 Oct;59(5):372-9. https:// doi.org/10.1093/tropej/fmt037

32. Albert J, Bogard J, Siota F, McCarter J, Diatalau S, Maelaua J, et al. Malnutrition in rural Solomon Islands: An analysis of the problem and its drivers. Matern Child Nutr. 2020 Apr;16(2):e12921. https://doi.org/10.1111/ $\underline{\operatorname{men} .12921}$ 